\title{
Development of a public geographical information system-based website to follow the impact of control activities of soil-transmitted helminths in endemic countries
}

\author{
Maria Paola Maurelli, ${ }^{1}$ Paola Pepe, ${ }^{1}$ Antonio Montresor, ${ }^{2}$ Denise Mupfasoni, ${ }^{2}$ \\ Martina Nocerino, ${ }^{1}$ Maria Elena Morgoglione, ${ }^{1}$ Vincenzo Musella, ${ }^{3}$ Giuseppe Cringoli, ${ }^{1}$ \\ Laura Rinaldi ${ }^{1}$ \\ ${ }^{I}$ Department of Veterinary Medicine and Animal Production, University of Naples Federico II, \\ CREMOPAR, WHO Collaborating Centre ITA-116, Naples, Italy; ${ }^{2}$ Department of Control of Neglected \\ Tropical Diseases, World Health Organization, Geneva, Switzerland; ${ }^{3}$ Department of Health Sciences, \\ University of Catanzaro Magna Graecia, Catanzaro, Italy
}

\begin{abstract}
Soil-transmitted helminth (STH) infections are among the most common neglected tropical diseases worldwide causing high morbidity and mortality rates in endemic areas. Preventive chemotherapy (PC) programmes and health education are recommended by the World Health Organization (WHO) to reduce the impact of STH in endemic countries. Following our role as WHO collaborating centre (WHO CC ITA-116), we have developed a WebGIS and a dataset to support PC programmes to monitor the impact of STH control. This vHealth presentation shows the potentiality of these tools in improving communication among WHO's regional and country offices, Ministries of Health, pharmaceutical industries and other partners.
\end{abstract}

\section{Background}

Soil-transmitted helminth (STH) infections, caused by round-

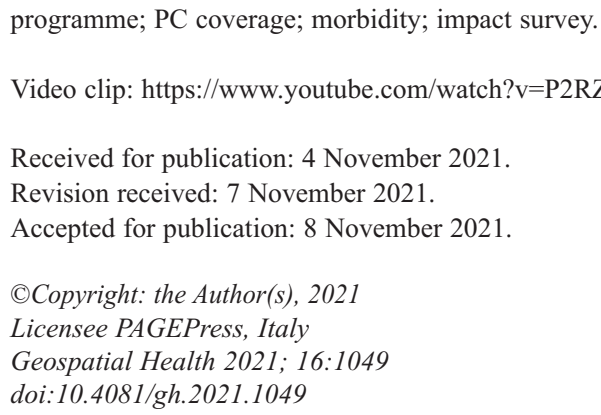
programme; PC coverage; morbidity; impact survey.

Received for publication: 4 November 2021.

Revision received: 7 November 2021.

Accepted for publication: 8 November 2021.

(C) Copyright: the Author(s), 2021

Licensee PAGEPress, Italy

Geospatial Health 2021; 16:1049

doi:10.4081/gh.2021.1049

Key words: Soil-transmitted helminths; preventive chemotherapy (PC)

Video clip: https://www.youtube.com/watch?v=P2RZE5UoSU0

This article is distributed under the terms of the Creative Commons Attribution Noncommercial License (CC BY-NC 4.0) which permits any noncommercial use, distribution, and reproduction in any medium, provided the original author(s) and source are credited. worms, hookworms and whipworms, are among the most common neglected tropical diseases (NTDs) worldwide. The World Health Organization (WHO) estimates that approximately 1.5 billion people are infected by STHs, with 2.0 billion at risk (WHO, 2020). The global burden of disease (GBD) amounts to over 3.3 million disability-adjusted life years (DALYs) (GBD, DALYs and HALE Collaborators, 2017; Turner et al., 2021) causing severe anaemia and hampering the cognitive and physical development of children leading to high morbidity and mortality rates in endemic areas (Marocco et al., 2020). The impact of STH infections is particularly high in low-income countries, especially in children, due to malnutrition, poor hygiene, low education and restricted access to health care (Hailegebriel et al., 2020). In addition, climatic changes and movement of people from high prevalence to non-endemic areas may increase the transmission of STH (Steverding, 2020). To reduce STH prevalence and morbidity, preventive chemotherapy (PC) strategy and health education, particularly targeted at pre-school and school age children (pre-SAC and SAC), are recommended (Montresor et al., 2020). Achieving and maintaining elimination of STH morbidity by 2030 are the main goals of WHO's programme as reported in the NTD 20212030 roadmap (WHO, 2020).

The terms of reference of our WHO collaborating centre for diagnosis of intestinal helminths and protozoa (WHO CC ITA116) based in Naples (Italy) are aimed to support endemic countries in monitoring PC programmes by: i) identifying countries in need of impact surveying; ii) following changes in the STH epidemiology (prevalence and morbidity) aiming at reduction in drug needs; and iii) indicating global, country and regional trend during PC implementation. For this reason, we have developed a WebGIS (www.whocc.ita116.unina.it) which includes a dataset for following the impact of STH control in the six WHO regions: Africa (AFRO), Americas (PAHO), South-East Asia (SEARO), Europe (EURO), Eastern Mediterranean (EMRO), Western Pacific (WPRO). In the accompanying video we show the potentiality of the tools proposed to facilitate communication among WHO's Department of Control of Neglected Tropical Diseases (WHO/NTD) and regional and country offices, Ministries of Health and pharmaceutical companies. The website also includes high-accuracy dynamic maps that are continuously updated based on data exclusively provided by WHO official channels. Any suggestion and update from the stakeholders listed above are more than welcome to improve the website. 


\section{Database development}

WHO has identified 87 STH-endemic countries in need of PC and six having achieved elimination, i.e. Burkina Faso, Niger, Mali, Maldives, French Polynesia and Malaysia. For each country we extracted data primarily from the standardized reports provided by WHO/NTD:

i) Joint Reporting Form (JRF) developed by WHO to facilitate the annual reporting of the drug coverage by NTD control programmes. JRFs consists of an Excel file with tags for each group of parasites targeted (STH infections, schistosomiasis; lymphatic filariasis and onchocerciasis); each at-risk group (pre-SAC, SAC, adult) for each endemic district/region/country (Montresor et al., 2020).

ii) Epidemiological Data Reporting Form (EPIRF) developed by WHO to collect epidemiological data on all diseases targeted from countries receiving donated anthelminthic drugs including type of survey; number of rounds of PC delivered prior to the survey; survey date; number of people examined and testing positive for each STH species and overall (Mupfasoni et al., 2019). From each EPIRF file we extract: year; target population; prevalence of each STH species; number of people examined and positive and intensity of infection.

The database also comprises data from a review of the available scientific literature from 2000 to 2020, including STH survey(s); official report(s) of national NTD plans; report(s) of Ministry of Health; the Weekly Epidemiological Record (WER) published annually for each NTD. Country-specific epidemiological data are collected also from the online Expanded Special Project for Elimination of NTDs (ESPEN) (espen.afro.who.int) and the Global Atlas of Helminth Infections (GAHI) (thiswormyworld.org), both with open-access availability.

Data obtained from the different sources are analysed and entered in a Microsoft access file.

Generally, data of any STH species in EPIRFs refer to the schools or villages grouped at administrative level 2 (i.e. district level) and where not possible at level 1 (i.e. region level) or level 0 (i.e. country level). All information needed is summarized and re-calculations are performed of each and any STH prevalence, including the prevalence of moderate and high intensity infections (MHI) accounting for the total number of people examined.

\section{Maps development}

Three different data maps (Progress of implementation; Impact of intervention on STH prevalence; and Impact of intervention on STH morbidity) were developed using the ArcGIS Pro 2.7 software (Environmental Systems Research Institute, Inc., Redlands, CA, USA). Each set contains a global dynamic map showing the full view with an interactive display that permits moving among the six regions and zooming into the different countries. It is easy to distinguish the countries in PC (coloured) from those that do not (grey). For all countries in PC (Progress of implementation maps), a flag with available information on endemicity and the number of people at risk is shown. In addition, static maps have also been developed to provide a detailed display of the six WHO regions including the smallest islands.

\section{Progress of implementation maps}

The progress of implementation of PC for STH is assessed analysing the national drug coverage (DC) reported in the last five years available (2015-2019). Since the conditions for each country are different, it is difficult to assign a precise period to continue PC for each one. To ease this problem, a formula has been prepared evaluating equally all the countries as described below. Regional and country offices reviewed the data obtained.

In order to monitor the effective implementation for each country a score from 0 to 3 is assigned to each annual percentage of DC (Table 1). The value attributed is calculated according to the following equation:

$(a / b) / \beta$

where $a$ is the sum of intervention values (Table 1 ); $b$ the number of interventions carried out; and $\beta$ the correction factor ( 5 years $/ b$ ). Based on values obtained, each country received a colour indicating their status (Figure 1). At this point, 10 countries show 'incomplete DC'; 37 are 'in need of implementing STH PC intervention'; 22 'in need of impact survey'; and 16 have completed the impact survey. Data were not yet available for eight countries.

\section{Progress on the two impact maps}

Endemic countries are invited to evaluate their progress with a survey (after at least five years of PC intervention) which provides results of the impact of the intervention in terms of percentage reduction in any STH and MHI. Indeed, these indicators are fundamental to assess whether the goals of STH programmes are achieved, i.e. any STH and MHI <2\% (Montresor et al., 2020).

\begin{tabular}{ll}
\hline Score range & Category assigned \\
\hline $0.0-0.4$ & Not started/not $100 \%$ geographic coverage \\
$>0.4-0.8$ & Need of 4 additional years of $\mathrm{PC}$ \\
$>0.8-1.0$ & Need of 3 additional years of PC \\
$>1.0-1.3$ & Need of 2 additional years of PC \\
$>1.3-1.6$ & Need of 1 additional year of PC \\
$>1.6-2.0$ & Need of impact survey \\
$>2.0-3.0$ & Full impact survey done/full impact survey done and PC stopped \\
\hline
\end{tabular}

Figure 1. Score results to assign a colour on 'Progress of implementation maps'. PC, preventive chemotherapy.

Table 1. Intervention value assigned to each percentage of drug coverage.

Drug coverage (\%) Intervention (value level)

\begin{tabular}{ll}
$\geq 0$ to $\leq 40$ & 0 \\
$\geq 41$ to $\leq 74$ & 1 \\
$\geq 75$ to $\leq 89$ & 2 \\
$\geq 90$ to $\leq 100$ & 3 \\
\hline
\end{tabular}


In the Impact of intervention on STH prevalence maps, shades of brown are assigned to countries (no. 28) having completed the evaluation and provided the data based on the STH prevalence calculated in the latest surveys. Any STH prevalence levels considered were: STH $>50 \%$; STH $\geq 20 \%-<50 \%$; $\geq 10 \%-<20 \%$; $\geq 2 \%$ $<10 \%$ and STH $<2 \%$. So far, three countries show a STH prevalence $>50 \%$; eight $\geq 20-<50 \%$; eight $\geq 10 \%-<20 \%$; one country $\geq 2 \%$ - $<10 \%$; and eight countries $<2 \%$.

In the Impact of intervention on STH morbidity maps, shades of pink are assigned to the countries (no. 24) having completed the evaluation survey and provided the data based on the MHI prevalence calculated in the latest surveys. MHI levels considered were: $\mathrm{MHI}>2 \% ; \mathrm{MHI} \leq 2 \%$ and $\mathrm{MHI}=0 \%$. So far, six countries show a $\mathrm{MHI}>2 \% ; 15 \mathrm{MHI} \leq 2 \%$; and three $\mathrm{MHI}=0 \%$. Further implementations of these maps might be done considering also other data/parameters/indices for populations at risk (e.g. pregnant women) and STH morbidity (e.g. anaemia).

\section{Tables and graphs}

On the website we also provide:

- 'Flag tables' reported under global view of each map, summarizing the categorization of the countries for each dataset.

- Summary tables for each country containing different information, e.g. number of people in need of treatment; year of PC start; and number of epidemiological units available for baseline and follow up estimation.

- Graphs on available prevalence for each STH species and overall pre and post intervention for each country.

- Graphs on available MHI for each STH species and overall pre and post intervention for each country.

\section{Limitations}

The impact data for some countries are only from an area that frequently is the most covered from the PC intervention and not representative of the whole country. Therefore, to avoid misunderstanding, in the 'Flag tables' we asterisk the countries where data are not obtained from a national survey.

The situation of each endemic country is schematically presented in a single category for each dataset, however, it is not possible to classify in this way large countries like India, where each region shows different progresses in implementation and impacts. Since, a sub national map and more detailed data would be necessary to represent adequately this situation, we left India without classification in meantime to collect more detailed information.

\section{Overall aim}

Dynamic maps visualizing the evolution of impact of PC programmes should be an effective tool for following the progress of the control programme globally, improving the coordination among WHO's local authorities and partners as well as identifying countries needing impact surveys. Information on the impact of the programme is essential to document the elimination of STH morbidity, the global reduction of prevalence levels and the reduction of needs anthelminthic drugs, all among WHO's main goals by 2030 (Montresor et al., 2020).

\section{Description of the software used for producing the video clip}

- The software platform ArcGIS Pro 2.7 (Environmental Systems Research Institute, Inc., Redlands, California, USA) was used to generate all the interactive maps based on the open-source world countries shapefiles.

- The main results of the work, commented by the voice of the authors, have been displayed using Microsoft PowerPoint 2019 (Microsoft Corporation, Redmond, WA, USA) and inserted in a short video clip recorded using the free and Open Broadcaster Software (OBS) Studio 27.0.1.

- The video clip is shared on the online video sharing platform YouTube, at: https://www.youtube.com/watch?v=P2RZE5UoSU0

\section{References}

GBD 2016, DALYs and HALE Collaborators, 2017. Global, regional, and national disability-adjusted life-years (DALYs) for 333 diseases and injuries and healthy life expectancy (HALE) for 195 countries and territories, 1990-2016: a systematic analysis for the Global Burden of Disease Study 2016. Lancet 390:1260-344.

Hailegebriel T, Nibret E, Munshea A, 2020. Prevalence of soiltransmitted helminth infection among school-aged children of Ethiopia: a systematic review and meta-analysis. Infect Dis Res Treat 13:1-14.

Marocco C, Tediosi F, Bangert M, Mupfasoni D, Montresor A, 2020. Estimated need for anthelminthic medicines to control soil-transmitted helminthiases in school-aged children, 20202030. Infect Dis Poverty 9:48-58.

Montresor A, Mupfasoni D, Mikhailov A, Mwinzi P, Lucianez A, Jamsheed M, Gasimov E, Warusavithana S, Yajima A, Bisoffi Z, Buonfrate D, Steinmann P, Utzinger J, Levecke B, Vlaminck J, Cools P, Vercruysse J, Cringoli G, Rinaldi L, Blouin B, Gyorkos T W, 2020. The global progress of soiltransmitted helminthiases control in 2020 and World Health Organization targets for 2030. PLoS Negl Trop Dis 14:e0008505.

Mupfasoni D, Bangert M, Mikhailov A, Marocco C, Montresor A, 2019. Sustained preventive chemotherapy for soil-transmitted helminthiases leads to reduction in prevalence and anthelminthic tablets required. Infect Dis Poverty 82:82-92.

Steverding D, 2020. The spreading of parasites by human migratory activity. Virulence 11:1177-91.

Turner HC, Stolk WA, Solomon AW, King JD, Montresor A, Molyneux DH, Toor J, 2021. Are current preventive chemotherapy strategies for controlling and eliminating neglected tropical diseases cost-effective? BMJ Glob Health 6:e005456.

World Health Organization, 2020. Soil-transmitted helminth infections. World Health Organization, Geneva, Switzerland. Available from: https://www.who.int/news-room/fact-sheets/ detail/soil-transmitted-helminth-infections maps'. PC, preventive chemotherapy. 